\title{
Innovative manufacturing technologies for the disassembly of consumer goods
}

\author{
E Uhlmann, F Elbing and $\mathbf{J}$ Dittberner* \\ Institute for Machine Tools and Factory Management, Technical University Berlin, Berlin, Germany
}

\begin{abstract}
Ecological harmless disposal of used technical consumer products will become mandatory for producers and importing companies. This disposal policy will focus on product and material loops; used products will be disassembled and the parts and materials then recycled. Owing to environmental and legislative reasons, the importance of disassembly as a step in the process of recycling is steadily rising. The article presents developed technologies and tools for the disassembly of consumer goods. The aim is to recover materials and reusable components within a semiautomatic pilot disassembly system. Different destructive processes were optimized to disassemble washing machines.
\end{abstract}

Keywords: ecological disposal, disassembly of consumer goods, recycling

\section{INDUSTRIAL DISASSEMBLY}

Not long ago, a product life started with assembly and ended with disposal. Today, in times of increasing waste quantity and decreasing natural resources, life cycles are invented in which materials are reprocessed or reutilized and components are reused [1] (Fig. 1). Old technical consumer products are disassembled. These old products are called disassembly goods or disassembly objects.

Disassembly is a major operation in the recycling of used technical products, but disassembly is not as highly automated as assembly yet. The standard of automation for disassembling is still very low and therefore dismantling is often performed manually $[2,3]$. Employment is slow and cost intensive, especially in the highly industrialized parts of the world such as Europe. Hence recycling is more economic by automated disassembly. On the other hand, the human worker is the most flexible tool available. When confronted with the high variety of types of technical product and the state that they are in after use, it is clear that a large amount of information has to be considered when choosing disassembly tools, processes and strategies.

The conclusion is that flexibility and speed are two main requirements for industrial disassembly. At the Technical University Berlin the Collaborative Research

\footnotetext{
The MS was received on 6 October 2003 and was accepted after revision for publication on 28 April 2004.

*Corresponding author: Institut für Werkzeugmaschinen und Fabrikbetrieb, Technische Universität Berlin, Pascalstraße 8-9, D-10587 Berlin, Germany.
}

Centre 281 (CRC281) works on basics, tools and processes for disassembly as well as on its planning and implementation. The objective is to increase the effectiveness and to minimize the costs of disassembly through optimized disassembly methods. It begins at the product planning phase and does not end at the actual technical disassembly process. This paper presents a general introduction of the four project areas of the CRC281 and focuses on the examinations of subproject dealing with the development of processes and tools for the separation of insoluble connections.

\subsection{Collaborative Research Centre 281}

Sustainability is a prerequisite for future development. Much research is carried out to obtain sustainable designing methods and manufacturing processes. Often, newly developed products are designed for material and product cycles, but these are future trends. Today there are products at the end of their life, which were not intended for reuse or reutilization but for disposal. They were not designed for disassembly and, since disassembly is not necessarily the inversion of assembly, innovative disassembly technologies have to be found.

At the Technical University Berlin and the Berlin University of Arts, the CRC281 disassembly factories for the recovery of resources from product and material cycles promoted by the Deutsche Forschungsgemeinschaft investigates industrial disassembly. In 1995 the project was started and it was granted a final 3 year term in 


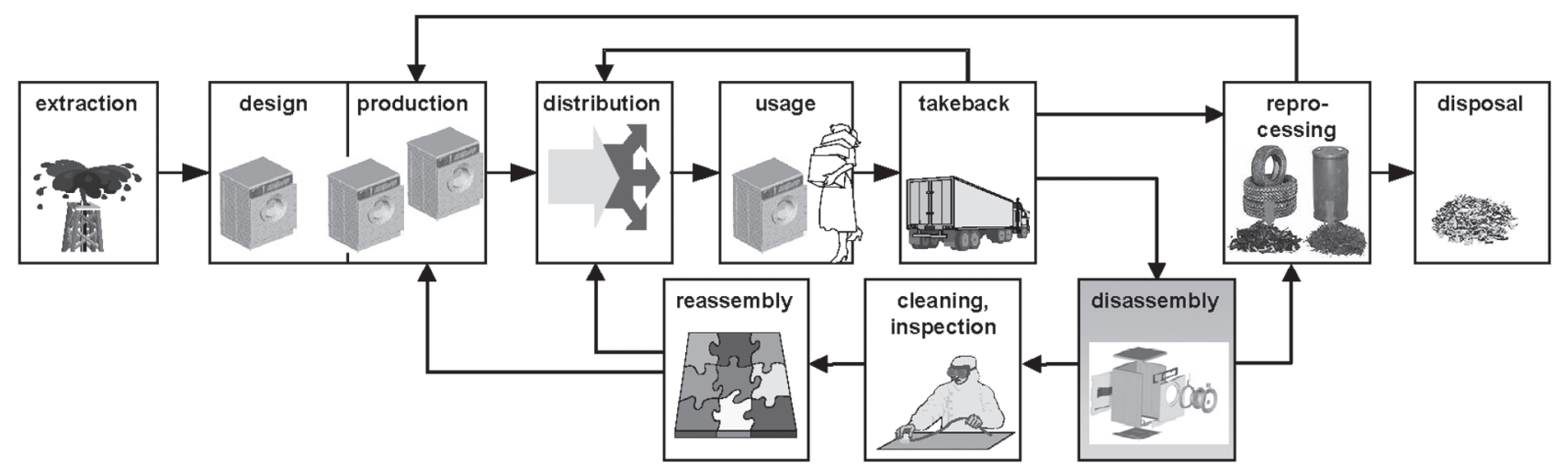

Fig. 1 Disassembly within a product's life cycle

2003. It is a collaboration of nine institutes from two universities.

The centre is divided into four different project areas, which deal with different aspects of disassembly [4] (Fig. 2). Each project area is divided into three to six subprojects, and 21 research engineers guide an overall of 16 subprojects.

Project area A, tools and processes, invents and optimizes single disassembly techniques and tools as well as a pilot disassembly system. Project area B deals with the logistic problems of collection and distribution in disassembly and describes ways to implement the disassembly factory in the environment. Project area $\mathrm{C}$ determines the value of a disassembly product and plans disassembly. Project area D designs disassemblyfriendly products and facilitates disassembly. In the following, an overview of the project areas is given. The Web pages www.sfb281.de contain more detailed information.

\subsubsection{Tools and processes}

In order to control and economically to optimize disassembly processes, a basic knowledge of these processes is required. Project area A delivers these elementary processes as well as tools, production facilities, information and sensor systems for disassembly [4]. Within the research period 2001-2003 the goal of project area A was to develop highly flexible, adaptable and modular processes and tool concepts for different life cycle scenarios. The research on cutting processes, information, sensor and disassembly systems had to be combined

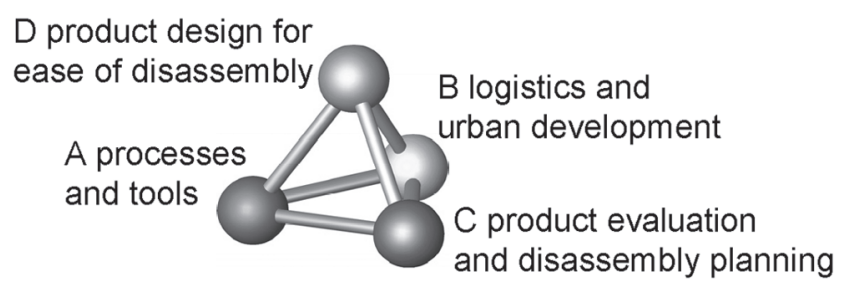

Fig. 2 Project areas of the CRC281 and verified within a pilot disassembly system. The overall focus is on advantages, disadvantages and restrictions of processes and tools concerning flexibility, modularity and adaptability.

Challenges for further investigations of disassembly processes and tools include fast, safe, ecological and economical disassembly of structural parts and materials by destroying them. As an example the separation of washing machine housings is tested in order to achieve fast access to valuable components.

New modular tools for manual and automated use are developed to disconnect joints and to handle disassembly objects. This is realized by the generation of new acting surfaces. Tools developed during the first and second research periods are integrated into a modular construction kit as functional units.

Further process and tool developments, e.g. desoldering, are related to non-destructive disassemblies in order to preserve component quality for reuse. A concept for clamping technologies for disassembly combines clamping and handling of different products. Within the project area, a cleaning technology with laser and dry ice blasting reuse and further use of disassembly products is developed.

The life cycle unit collects life-cycle-related data in order to support disassembly processes. Detailed information about product and component quality is available throughout the entire life cycle. Product information about the steering of disassembly and reassembly are equally gathered.

The development of processes and tools, security directives, information systems, effectors and sensors is the first step in the direction of automation. The product area is divided into six subprojects, which deal with the separation of insoluble connections, shape-independent end effectors for non-destructive disassembly, flexible clamping technology, protection of labour and safety precautions, cleaning technologies, sensor-guided processes and realization of a pilot disassembly system. Within the disassembly system the interplay of the developments of the other subprojects is tested. Disassembly operations and sequences are put together 
and optimized. Disassembly objects are up-to-date washing machines, used car engines and a disassemblyfriendly washing machine designed in the project.

\subsubsection{Logistics and urban development}

It is necessary to integrate logistics, disassembly factories and urban structures in order to achieve closed product and material loops. Project area B develops logistical disassembly systems in close relation to the pilot-disassembly system of product area A, as well as other in-house logistic systems. The object is to match external logistics with the logistics of customers as well as with suppliers. This requires a certain organization, which has to be designed and installed prior to realization [4]. Questions of collection and storage of disassembly goods are answered, and procedures optimized. In addition, scenarios of the necessary infrastructure are developed as well as methods to integrate disassembly factories into urban or rural environment. One emphasis is the disassembly-friendly construction of the disassembly factory itself. The project is divided into three subprojects which deal with the process-oriented development of logistical systems, the creation of a regional network of disassembly factories and the interaction between disassembly factory and city.

\subsubsection{Product evaluation and disassembly planning}

A substantial requirement of disassembly is the investigation of the individual product. Planning and realization of disassembly facilities are strongly influenced by uncertainties, which result from usage of products. To meet resulting requirements the systems have to be flexible and offer an on-line control potential reaching far further than any given system available today.

Information relevant for disassembly, such as experience gained from previous efforts, general product information and the usage-related condition of the product, must be considered together. The disassembly process of an individual product cannot be precisely predicted ahead of time and must be planned just prior to the start of the process or even during the process. This situation results from different conditions in which a product may be dependent on the influence of usage.

Evaluation of disassembly relies heavily on the difference between costs and benefits. On the side of costs it is most important to give values to a wide range of different items such as emissions, resource consumption or social costs. To evaluate items as social costs, it becomes necessary to find appropriate cost-accounting systems. As one result, the system found can help to improve existing products and, additionally, the quality of new products.

Project area $\mathrm{C}$ is divided into three subprojects, which deal with methods of assessing recycling suitability and computer aided disassembly planning and control as well as with the economic and ecological course of action [4].

\subsubsection{Product design and ease of disassembly}

Disassembly efficiency will be increased if design for ease of disassembly requirements such as reusable component architecture or pure material use are met. Emphasis lies on joints. It is demanded not only that joints offer accessibility for tools but also that they are easy to disassemble. The deduction of design criteria provides a great deal of support for assessment during the design stage. To predict end-of-life product conditions, simulation tools are needed which require an information network that provides and receives information from producers, users and recyclers.

Project area $\mathrm{D}$ is divided into four subprojects for the product, with the selection, embodiment and arrangement of fasteners, with design guidelines for structures and joining components, with simulation tools for a disassembly-adequate product design and with the disassembly-oriented information-technological infrastructure [4].

\section{SEPARATION OF INSOLUBLE CONNECTIONS}

Within project area A the subproject A1, separation of insoluble connections, develops processes and tools for destructive disassembly $[\mathbf{4}, \mathbf{5}]$. The task was approached from two different points of view. One was the classification of components and joints. The other was the categorization of existing manufacturing technologies.

Disassembly products consist of components and joints. Components are made of sheets, poles or more complex workpieces. Joints are rivets, screws, welds or forming connections. Ideally, to disassemble a consumer product all joints have to be dismantled. Often this is not possible. This may result from the connection itself as with welded joints, or from external influences on it such as corrosion or damage.

These connections have to be destroyed and are called insoluble. Also it might prove more effective not to dismantle connected parts but partly to destroy one part to obtain fast access to downstream parts that may be reusable.

Plasma arc cutting, drilling, water jet cutting, abrasive cutting, splitting and shearing are examined, as well as a combination of these with a cryogenic pre-treatment with liquid nitrogen. One emphasis in the research lies on the minimization of side effects that might influence the functionality of reusable parts. Some processes implement forces on the parts to be disassembled and thus cause unwanted deformations. Other processes cause a spray of sparks or material particles that damage or contaminate other parts and hence the necessary reconditioning has to be extended. Another emphasis is to understand how to use the processes for disassembly properly, in a way that is highly 
flexible and cost effective. Often these goals contradict each other. Then a practicable compromise has to be found.

Some of the tested processes are integrated in the pilot disassembly system. In it the first example for a disassembly object was a washing machine. There the first task for the destructive processes was to open up the case of the washing machine and to gain fast access to reusable components such as the motor, the pump or the valve unit.

Most cases for washing machines are made of steel. Others are made of stainless steel, and a few of aluminium. The coatings used are enamel paints, dry lacquers or two-component lacquers. Thus different combinations of material and coating were used for the examinations. The goal is to cut sheets out of each wall of the housing. The sheets have to be as large as possible to allow easy access to inner parts. Thus a frame is left to support the swinging system. In the process of opening a housing, long cuts have to be produced. Adequate processes for this purpose were plasma arc cutting, water jet cutting and abrasive cutting.

Inside a washing machine, other non-reusable parts were identified for destructive disassembly. Bars, wire and point-shaped connections were among these. It is possible to destroy these parts and joints by plasma arc cutting, water jet cutting and abrasive cutting, too. However, compared with the small size of the object they cause a high amount of different emissions, sparks and by-products. Therefore dividing processes such as shearing and splitting were tested. A hydraulic blade cutter was used to shear bars and wire. An adjusted chipping hammer splits screw heads and rivet heads from the base bodies. Contrary to the other processes, the amount of used energy and the geometry of blades and acting effectors had to be optimized.

In the following, some of the various examinations on plasma arc cutting and water jet cutting are described.

\subsection{Plasma arc cutting}

For the disassembly of washing machines, specific disassembly sequences were elaborated for each type. The washer case is separated by means of plasma arc cutting in such a way that a frame remains, to which the inner components are attached. The aim of this disassembly step is to reach the inner components and assemblies as rapidly and cost-effectively as possible. A robotic plasma arc cutting device, PA-S 45 CNC by Kjellberg, Finsterwalde, Germany, was used. The installation works with a transmitted plasma arc at cutting amperages of 40-130 A, a cutting voltage of $160 \mathrm{~V}$ and a cutting power of $20 \mathrm{~kW}$. In the course of technical investigations, a qualitative gas analysis was carried out as well as process optimization with regard to the maximum cutting speed and a completely cut-through workpiece, minimum mechanical and thermal component damage and minimum removal particle mass [6].

The maximum cutting speed was obtained in experiments with the standard experimental set-up by Tagucci, during which the parameters (cutting amperage, cutting gas, workpiece material and material thickness) were varied. The cutting gases tested were air, $\mathrm{Ar}-\mathrm{H}_{2}$ and $\mathrm{Ar}-\mathrm{H}_{2}-\mathrm{N}_{2}$. The maximum cutting speeds could be obtained with air. Irrespective of how rapid the cut with air may be, there is a high emission of removal particles. With increasing cutting amperage the maximum cutting speed increases too. At a cutting average of $125 \mathrm{~A}$ a maximum cutting speed of $12 \mathrm{~m} / \mathrm{min}$ can be achieved. The cutting amperage and the cutting gas with a minimum removal particle mass were determined.

Figure 3 displays the removal particle mass as a function of the cutting amperage in the case of plasma arc cutting of a workpiece similar to the washer case of the Bosch V454 type. After optimization the particle emission during plasma arc cutting of the washer case could be reduced by 95 per cent compared with the initial setting, which employed a cutting amperage of $125 \mathrm{~A}$ and air as the cutting gas. The conclusion is that the removal particle mass is almost irrespective of the cutting speed in the case of a defined separating cut and that it is only minimal when an $\mathrm{Ar}-\mathrm{H}_{2}$ cutting gas and a cutting amperage of $75 \mathrm{~A}$ are applied. A disassembly time of $3 \mathrm{~min}$ is calculated for the industrial disassembly of a complete washing machine case if optimized parameters are applied during the separation of the four lateral walls of the washer case. After that, the assemblies and components can be disassembled in a destruction-free manner.

Including the results of the above-described examinations the construction of the plasma arc plant and the periphery were altered and integrated into a hybrid pilot disassembly system. Owing to the high cutting speed, good automation and low cutting costs, plasma arc cutting was used in the pilot disassembly system to open washing machine cases from various suppliers. Further examinations took place on cutting poles and wires by plasma arc cutting.

\subsection{Water jet cutting}

A robotic abrasive water jet cutting device from Ingersoll Rand, Bad Nauheim, Germany, was used for the examination of water jet cutting in disassembly. Again metallic materials were processed. The maximum working pressure was $320 \mathrm{MPa}$, the average abrasive mass flow was $350 \mathrm{~g} / \mathrm{min}$ and an abrasive garnet mesh 80 was used.

The first focus is on the optimization of the maximum traverse rate at which a workpiece is cut through. Depending on the material and the workpiece thickness a model was built to predict the maximum traverse rate. Besides the material properties, the working distance, abrasive 


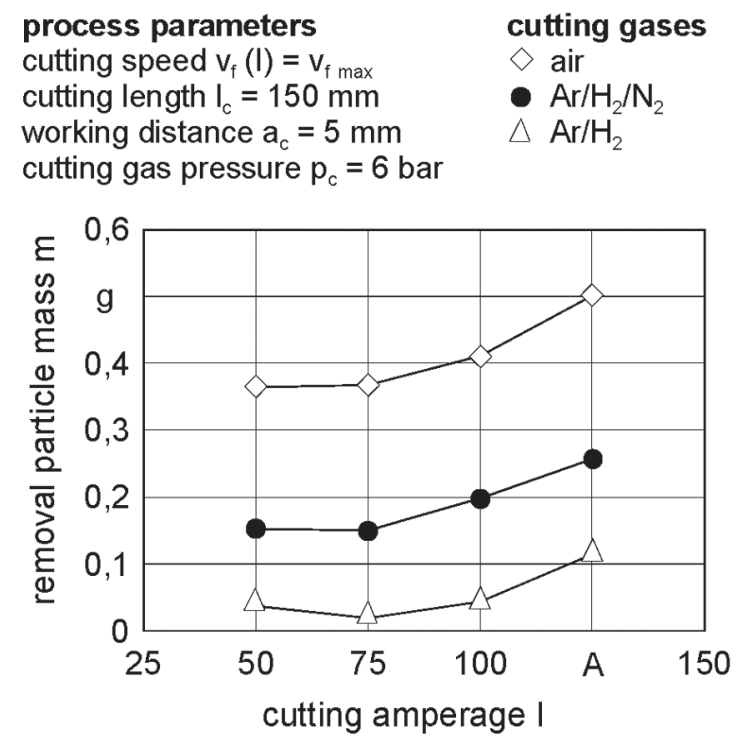

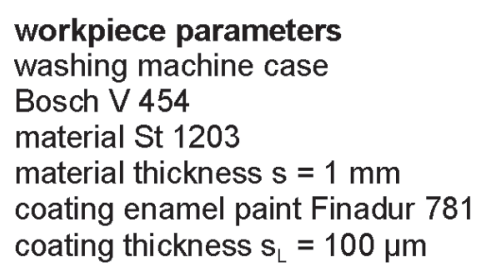

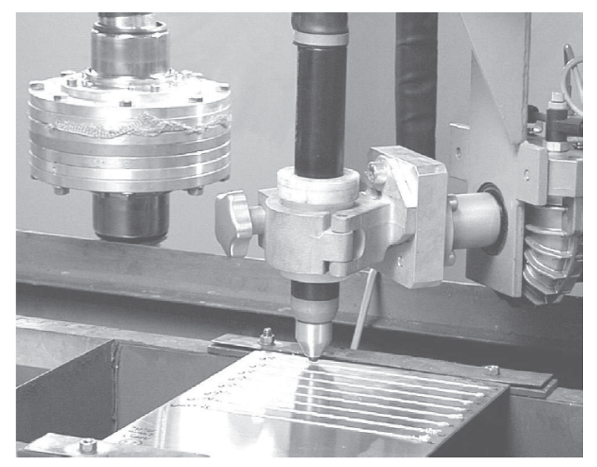

Fig. 3 Removal particle mass as a function of cutting amperage and cutting gas during plasma arc cutting of a washing machine case

mass flow and the working pressure quantify the traverse rate. It is highest at the maximum pressure and an abrasive mass flow of $350 \mathrm{~g} / \mathrm{min}$ [7].

Another task of the investigations on water jet cutting was to determine and explain the jet properties, and particularly the influence of the secondary jet on inner components and assemblies. If water jet cutting is used for three-dimensional machining, damages occur caused by the primary and the secondary jet. The primary jet is the jet prior to machining and the secondary jet exists behind the workpiece that is machined and ceases in infinity or when striking inner components. Within disassembly the chances are high that the secondary jet strikes an inner component or other assemblies. When the so-called secondary jet distance, the distance between the primary workpiece and inner components, is small, reusable parts are at risk of being damaged. Thus the objective is to minimize the energy of the secondary jet and to predict its influence on other components. The secondary distance is measured on a straight line, which runs vertically to the primary workpiece through the jet exit point.

With knowledge about the kerf geometry, it is possible to predict the depth of the damage from the secondary jet. Essentially, the damage of the inner components depends on the traverse rate and the secondary jet distance, which is the gap between the primary and secondary workpieces. Ideally, the damage inflicted by the secondary jet during cutting with the maximum traverse rate is zero. Thus the traverse rate must be seen in relation to the maximum traverse of the primary workpiece. As the traverse rate becomes closer to the maximum value, the secondary jet energy decreases and the possible damage is less.

The secondary jet spreads according to the same rules as a primary water jet. As a result, it is possible to minimize and predict the damage of inner components. With this knowledge, it is possible to set cutting strategies for disassembly, which avoid damage on reusable components.

The abrasive water jet was integrated into the disassembly process of standard washing machines to open the plastic lye container and to separate it from the washing cylinder made from stainless steel. Figure 4 shows the disassembly stages of a washing machine. It starts with the opening of the case, followed by the separation of the swinging system, which is then stripped from the weights, pump and motor. Afterwards the water jet opens up the lye container and the washing cylinder is removed.

\subsection{Conclusion}

The possibilities of using destructive separating manufacturing processes for the disassembly of old consumer products exist but are restricted. When used alone, there is always a side effect that might affect reusable parts. Measures have to be taken to neutralize the side effects. It is possible to remove contamination by cleaning. Damage can be repaired. To save workers and the environment from emissions, exhaust and filter systems have to be installed. All these measures cause disassembly costs to rise, either as a result of a more complex technical and mechanical equipment or by increasing the time for disassembly.

Another way to minimize those effects is to combine the processes with another operation. Already cryogenic pre-treatment with liquid nitrogen has been tested. By cooling material to a temperature of nearly $-200^{\circ} \mathrm{C}$, it is possible to embrittle the material. Thus lower forces occur while cutting material and it is possible to increase 


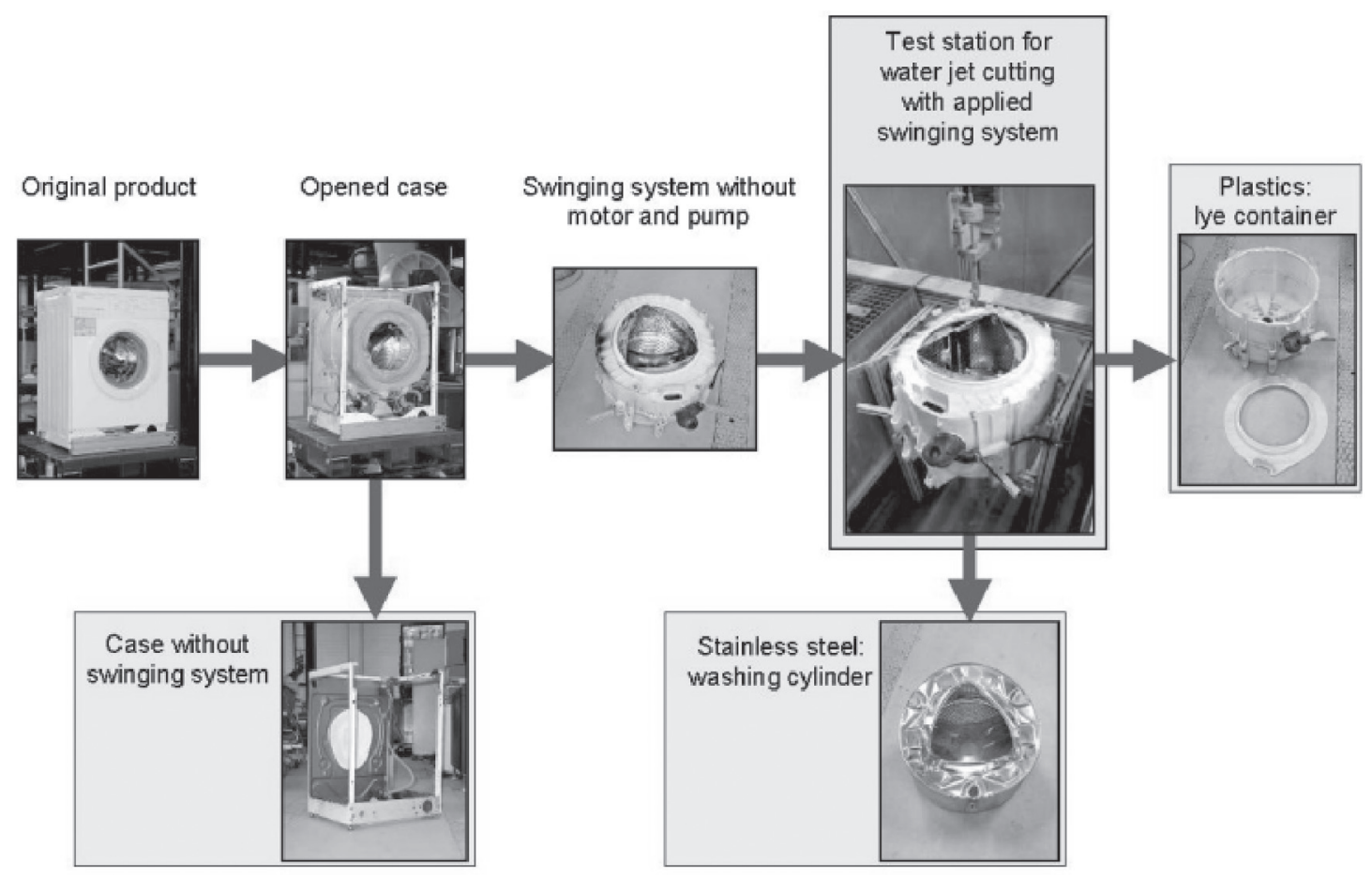

Fig. 4 Disassembly stages of a washing machine

the cutting speed. The time to split a screw head from a screw of $6 \mathrm{~cm}$ diameter was reduced from 8 to $3 \mathrm{~s}$ while the nitrogen application took about $2.5 \mathrm{~s}$.

Another aim within the subproject is to let the worker in disassembly know which process is the best for a task. Therefore a process selection system (PSS) was developed. All information about the examined processes is pooled in this system. The main objective of the selection system is to help to identify the most efficient process for a specific disassembly operation. Thus, an existing disassembly plan was assumed. Therefore, there must be knowledge about which part can be reused and how to gain access to it.

Figure 5 shows the environment in which the PSS is embedded. Industrial disassembly requires exact planning of the disassembly sequence for each product. For every single step of this sequence an adequate process has to be determined. By linking the processes it is possible to find the cheapest and fastest method for the whole sequence. The step is described by the kind of part, material, geometry, surface, further usability and its position. Within the PSS the information is compared with the collected technological data. A matching disassembly process is selected and written into the disassembly plan. In the following, the content and procedure of the PSS is described in detail.

The first piece of information that the system needs is which part has to be worked on and its specific features. Then, a practical working sequence has to be established. The recycling purpose for disassembled parts has to be defined. The user then has to decide which criterion, technological or economical, the output has to refer to.
Figure 1 shows the resulting selection sequence of the PSS. The single steps are described in the following.

As described above, it is possible to categorize the disassembly objects for destructive processing into sheets, joints and bars. Sheets are flat plates or formed threedimensional formations such as housings or cases. For destructive processing, the planes that create such a formation are taken as single parts.

Because of the very wide variety of existing joints they have to be subdivided. The user has to choose between

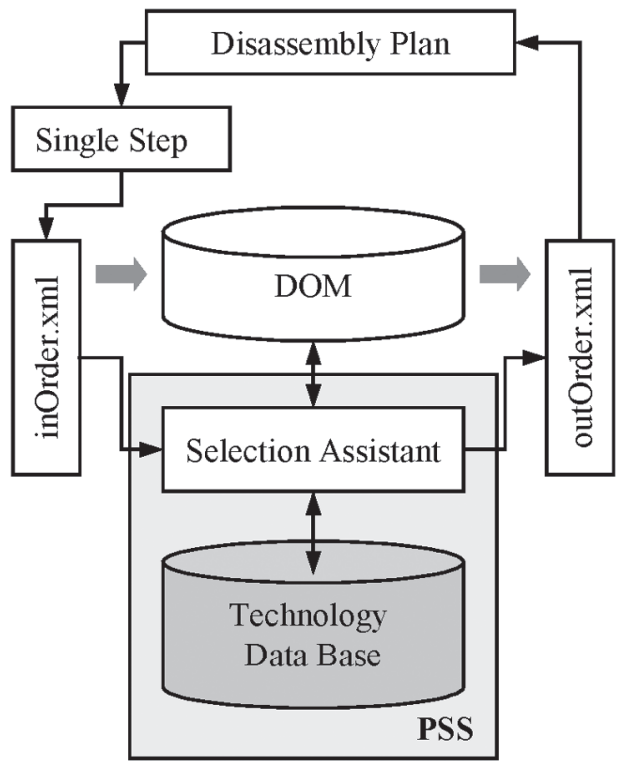

Fig. 5 Environment of the PSS (DOM, document object model) 
point, plane and linear connections. A bar has small cross-section compared with its length. Examples are poles, springs and wires.

It is possible to choose between geometrical, topographical and material features. The features are already categorized according to the results of optimization of the researched processes. For example, sheets are subdivided into sheets with thicknesses of $0.1-2.5 \mathrm{~mm}$, $2.5-5 \mathrm{~mm}$ and $5-10 \mathrm{~mm}$. Topographical features are macroscopic surface features such as fins, ribs or holes.

The user chooses the appropriate working sequence, which is a contour, a straight line or a point. This relates to the disassembly plan. Then, an enquiry is made whether the operation has to be executed once or many times. It is significant for the selection if a housing is connected to a supporting frame by 20 rivets or with one welding line, for example. The value of the part to be disassembled is estimated. Is it a reusable part or intended only for utilization? Reusable parts are more valuable. To gain high profit, disassembly processes have to have little influence on inner parts in order to minimize rework and costs.

Within this selection step the user chooses between different scenarios. It depends on the location where the work takes place and on whether manual or automated processes are preferred. The choice between different locations relates to whether the disassembly machinery is used stationary or mobile. When mobile machinery is used, not all researched processes are useful due to their extent and complexity. In addition, it is possible to pre-select processes and tools already existing at the user's location.

The user has the opportunity to specify the preferred criteria for the resulting selection sheet. It is possible to select whether the order of possible processes relates to the costs or to the rate. This feature is important, for example, if a single punctual connection has to be destroyed. A user might be interested in knowing which is the fastest method. If there is no preference between these two criteria, the system will arrange the processes according to the disassembly costs automatically.

In this selection step the user is given an output table with the variety of possible processes for the specific task. The processes are according to the selected criteria ordered. Moreover, the table contains secondary information about safety precautions and occurring secondary damage. The notes on safety give precaution instructions to follow, e.g. whether an exhaust or goggles are necessary. To summarize all prior selection steps the sheet additionally lists the given information.

\section{OUTLOOK}

The challenges for industrial disassembly are new life-cycle management laws, a growing recycling and maintenance industry, the handling of contamination and harmful chemicals in recyclable products, and the reuse of components from recyclable products. An approach to this field is made by the CRC281 disassembly factories. Focuses are on the disassembly of consumer goods and on the cleaning of components for reuse. The object is to develop processes and tools for fast and economical disassembly.

The described examinations show the independence of water jet cutting and plasma arc cutting from the material and working direction. The processes were compared with other processes such as drilling, abrasive cutting, shearing, splitting and a self-developed strikecutting tool. Plasma arc cutting is the most flexible and most rapid process for the disassembly of metallic consumer goods. Only when it comes to disassembling non-metallic components is water jet cutting even more effective; otherwise it is the second choice. The flexibility of these processes is their main advantage. The main disadvantage is the occurrence of only partly controllable and non-eliminable side effects. The approach of using processes that only have high process forces as a side effect on the disassembly object is very promising. Therefore other processes will be combined with cryogenic pretreatment. Also it is possible to use heat to lower the cohesion of a material. It is planned specifically to heat parts. This will be achieved by means of induction, friction, electricity, sound, flames, electric arc, etc. In the long run the examination will be the basis for the development of new destructive methods and selfdestructing connections.

In addition the heating processes themselves will be tested. The so-called melting processes belong to the manufacturing processes not yet examined.

All collected data and information will be added to the PSS. It will also be extended with information about non-destructive disassembly techniques and tools.

\section{REFERENCES}

1 Spath, D., Klimmek, M. and Tritsch, Ch. Technologies for efficient disassembling and dismantling of discarded technical products. In Proceedings of the 3rd International Seminar on Life Cycle Engineering, Zurich, Switzerland, 1996.

2 Scholz-Reiter, B., Scharke, H. and Hucht, A. Flexible robot-based disassembly cell for obsolete TV-sets and monitors. Robotics Computer Integrated Mfg, 1999, 15, 247-255.

3 Feldmann, K. and Meedt, O. Recycling and disassembly of electronic devices. In Life Cycle Modelling for Innovative Products and Processes (Eds F.-L. Krause and H. Jansen), 1996, pp. 233-245 (Chapman and Hall, London).

4 Seliger, G. (Ed.) Finanzierungsantrag 2001-2003. Sonderforschungsbereich 281 'Demontagefabriken zur Rückgewinnung von Ressourcen in Produkt- und Materialkreisläufen', Technical University Berlin, 2000. 
5 Uhlmann, E., Axmann, B. and Elbing, F. Fast disassembly of point forming and welded connections. In Proceedings of the 4th World Congress on Recovery, Recycling, Re-integration (R'99), Geneva, Switzerland, 1999, pp. 402-411.

6 Spur, G., Uhlmann, E., Elbing, F., Dittberner, J., Sundaresan, S. and Thantry, P. B. Flexible automatic disassembly for the recycling of consumer goods. In Advances in Manufacturing
Technology XIV, Proceedings of the 16th National Conference on Manufacturing Research, London, 2000, pp. 407-411 (Professional Engineering Publishing, Bury St Edmunds and London).

7 Uhlmann, E., Axmann, B. and Elbing, F. Model of kerf and simulation of damage in abrasive water jet cutting. Prod. Engng, 1999, 1(2), 51-54. 